EPJ manuscript No.

(will be inserted by the editor)

\title{
The $\mathrm{Z}=82$ shell closure in neutron-deficient $\mathrm{Pb}$ isotopes
}

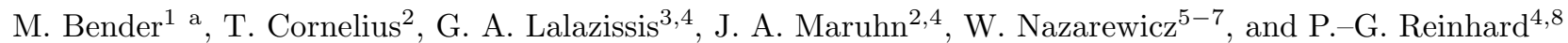 \\ 1 Gesellschaft für Schwerionenforschung, Planckstrasse 1, D-64291 Darmstadt, Germany. \\ 2 Institut für Theoretische Physik, Universität Frankfurt, Robert-Mayer-Strasse 8-10, D-60325 Frankfurt am Main, Germany. \\ 3 Department of Theoretical Physics, Aristotle University of Thessaloniki, Gr-54006 Thessaloniki, Greece. \\ 4 Joint Institute for Heavy-Ion Research, Oak Ridge National Laboratory, P. O. Box 2008, Oak Ridge, Tennessee 37831. \\ 5 Department of Physics and Astronomy, University of Tennessee, Knoxville, Tennessee 37996. \\ 6 Institute of Theoretical Physics, Warsaw University, ul. Hoża 69, PL-00681, Warsaw, Poland. \\ 7 Physics Division, Oak Ridge National Laboratory, P. O. Box 2008, Oak Ridge, Tennessee 37831. \\ ' 8 Institut für Theoretische Physik II, Universität Erlangen-Nürnberg, Staudtstrasse 7, D-91058 Erlangen, Germany.
}

October 232001

\begin{abstract}
Recent mass measurements show a substantial weakening of the binding energy difference $\delta_{2 p}(Z, N)=E(Z-2, N)-2 E(Z, N)+E(Z+2, N)$ in the neutron-deficient Pb isotopes. As $\delta_{2 p}$ is often attributed to the size of the proton magic gap, it might be speculated that reduction in $\delta_{2 p}$ is related to a weakening of the spherical $Z=82$ shell. We demonstrate that the observed trend is described quantitatively by self-consistent mean-field models in terms of deformed ground states of $\mathrm{Hg}$ and Po isotopes.
\end{abstract}

PACS. 21.10.Dr $-21.60 . \mathrm{Jz}-21.10 . \mathrm{Gv}-27.70 .+\mathrm{q}-27.80 .+\mathrm{w}$

\section{Introduction}

The weakening or "quenching" of spherical shell closures ' when going away from the valley of stability to weakly bound nuclei is a phenomenon of great current interest. It is now well established for neutron-rich $N=20$ and $N=28$ isotones. The study of excited states of nuclei around ${ }^{32} \mathrm{Mg}$ populated by $\beta^{-}$-decay [1] strongly indicates that neutron-rich $N=20$ nuclides are deformed. This is consistent with direct mass measurements which, at magic numbers 20 and 28 , do not yield the familiar drop in the two-neutron separation energy [2,3,4]. Energies and $B(E 2)$ transition probabilities obtained from Coulomb excitation of radioactive beams of nuclides around ${ }^{44} \mathrm{~S}$ demonstrate also that $N=28$ isotones below ${ }^{48} \mathrm{Ca}$ show strong collectivity in contradiction with a spherical shell closure [5]. Also, there are experimental hints from $\beta^{-}$decay studies of ${ }^{80} \mathrm{Zn}[6]$ and the systematics of $2^{+}$and $4^{+}$excitation energies in $\mathrm{Cd}$ and $\mathrm{Pd}$ isotopes [7] that the $N=50$ and $N=82$ shells are weakened when going towards neutronrich nuclei. Shell-quenching in neutron-rich systems has far-reaching consequences for astrophysics as it influences the $r$-process path [8].

The actual sequence of magic numbers in neutron-rich nuclei is strongly influenced by an increasing diffuseness of the neutron density, the closeness of the particle continuum, and/or the changes in the spin-orbit splitting [9.10].

a Present address: Service de Physique Nucléaire Théorique et Physique Mathématique, CP229, Université Libre de Bruxelles, B-1050 Bruxelles, Belgium.
For light neutron-rich $N=28$ isotones, this leads to an increased collectivity and even strong stable quadrupole deformation; see, e.g., refs. 11. 12, 13]. (For more discussion of shell structure of neutron-rich magic and semi-magic nuclei, the reader is referred to ref. [14 where a more complete literature review is given.)

The experimental signatures of "magicity" could sometimes be contradicting. Consider, e.g., the $N=40$ subshell in ${ }^{68} \mathrm{Ni}$. While it is clearly visible in the systematics of excited states [15,16], no sign of a shell effect is found when looking at two-neutron separation energies. Theoretically, this apparent inconsistency can be explained in terms of dynamic correlations beyond the mean field [17]. The calculated single-particle spectra in ${ }^{68} \mathrm{Ni}$ indeed show a $N=40$ shell. However, the sudden increase in collectivity in the adjacent isotopes obscures the picture when looking at isotopic energy differences because one now compares nuclei with different intrinsic structure. This example demonstrates that the various signatures of shell closures are not always equivalent.

All examples mentioned so far concern neutron shells. The situation seems to be different for protons. For light nuclei there is no indication that the proton shell closures fade away towards the proton drip line. The analysis of recent large-scale mass measurements of proton-rich nuclei around $Z=82$ at GSI [18] indicates a weakening of the proton shell gap in neutron-deficient $\mathrm{Pb}$ isotopes. This is found when looking at the binding-energy differences [19] and $Q_{\alpha}$ values [20]. It is the aim of this paper to 
M. Bender et al.: The $\mathrm{Z}=82$ shell closure in neutron-deficient $\mathrm{Pb}$ isotopes

analyze these data from a theoretical perspective using self-consistent mean-field models.

\section{Signatures of shell closures}

The notion of a shell closure comes from a mean-field description where one has full insight into the single-nucleon energies $\epsilon_{k}$ as eigenvalues of the single-particle Hamiltonian. A shell closure is associated with a large gap in the spectrum of $\epsilon_{k}$. It has to be stressed that $\epsilon_{k}$ are not equivalent to experimental single-particle energies $S_{k}$ which are measured as one-nucleon separation energies. To calculate those, one has to take into account residual interactions (such as pairing and coupling to low-lying vibrational modes), as well as rearrangement effects and the core polarization due to the unpaired nucleon; see, e.g., refs. 22,23.

Bunchiness of levels and the presence of gaps in the single-particle spectrum can be quantified in terms of the shell-correction energy 21]

$$
E_{\text {shell }}=\sum_{k} \epsilon_{k}-\int \mathrm{d} \epsilon \epsilon g(\epsilon)
$$

where $g(\epsilon)$ is a smoothed level density. $E_{\text {shell }}$ is large and negative for magic nuclei because there the discrete sum has a particularly low value while the smoothed expression varies only slowly with mass number. It is to be noted that $E_{\text {shell }}$ is a measure of the deviation of the actual level density at the Fermi energy from the smoothed level density. For very heavy systems beyond lead, the large level density inhibits pronounced shell gaps, and, yet, a dilution of levels suffices to produce shell stabilization without magic gaps; see ref. 24]. Shell corrections discussed in this study were calculated using the Green's function approach of ref. 25]. The advantage of this new method is the proper treatment of unbound states which appear close to the Fermi level in weakly bound (proton-rich or neutron-rich) nuclei.

Neither $\epsilon_{k}$ nor $E_{\text {shell }}$ are directly measurable quantities. A quantity which is accessible from mass systematics is the so-called two-proton shell gap

$$
\delta_{2 p}(Z, N)=E(Z-2, N)-2 E(Z, N)+E(Z+2, N),
$$

which is the approximation to the second derivative of the nuclear binding energy. Let us assume that the singleparticle energies do not change within the three isotones considered in Eq. (2), and that the change in the total binding energy comes from the variation of occupations around the Fermi surface. In such a case, Koopman's theorem states that $\delta_{2 p}$ should represent twice the gap in the corresponding single-particle spectrum. This requires, however, that no dramatic rearrangements happen among the three nuclei involved in $\delta_{2 p}$. For many nuclei, such rearrangements are small and $\delta_{2 p}$ is a good representation of a shell gap. But this interpretation does not hold in situations where adding or removing just two nucleons induces a substantial change of the mean field. In spite of this weakness, it is worth noting that binding-energy differences involving even-even nuclei only - like $\delta_{2 p}$ and, to some extent, $Q_{\alpha}$ - provide a cleaner signal and spectral properties than the binding energy expressions connecting to the neighboring odd-mass systems. The double difference $\delta_{p}$ with odd-mass neighbors mixes shell gap, and possibly rearrangement effects, with pairing effects, which surely imply a dramatic rearrangement of the pairing field by blocking.

Other experimental signatures of magic numbers are excitation energies of vibrational states and the associated electric transition rates. The gap in the single-particle spectrum sets the scale for the lowest excitations. Peaks in the systematics of the lowest $2^{+}$states in even-even nuclei reflect the stiffness of the potential energy surface which is large in closed-shell nuclei. This signature is somewhat masked by the residual interaction which shifts the excitation energy considerably below the lowest particlehole (or two-quasiparticle) energy. Compared to bindingenergy differences, the data on vibrational states have the advantage that they do not mix information from different nuclei. On the other hand, calculations of collective excitations require the use of techniques which go beyond the mean-field approach; hence the information on magic gaps is very difficult to extract.

\section{Theoretical framework}

We investigate the stability of the $Z=82$ shell in the framework of the self-consistent mean-field theory, either non-relativistic, using Skyrme interactions (SHF), or the relativistic mean-field (RMF) approach. As earlier studies have shown that parameterizations of the models often differ when extrapolated to weakly bound systems, we choose a sample of representative parameterizations which all give a very satisfactory description of stable nuclei but differ in details. Namely, we used the Skyrme interactions SkP [26], SLy6 [27], SkI3, and SkI4 28]. In the relativistic calculations, we employed NL3 [29] and NL-Z2 [30] parameterizations. The force SkP has effective mass $m^{*} / m=1$ and was originally designed to describe the particle-hole and particle-particle channel of the effective interaction simultaneously. The forces SLy6, SkI3, and SkI4 stem from recent fits which already include data on exotic nuclei. Both SkP and SLy6 use the standard spin-orbit interaction. The forces SkI3/4 employ a spinorbit force with modified isovector dependence. SkI3 contains a fixed isovector part analogous to the RMF, whereas SkI4 is adjusted allowing free variation of the isovector spin-orbit term. The RMF force NL-Z2 is fitted in the same way as SkI3 and SkI4 to a similar set of observables. Pairing is treated within the BCS approximation using a zero-range delta force with the strength adjusted for each mean-field parameterization as described as in ref. [31]. 


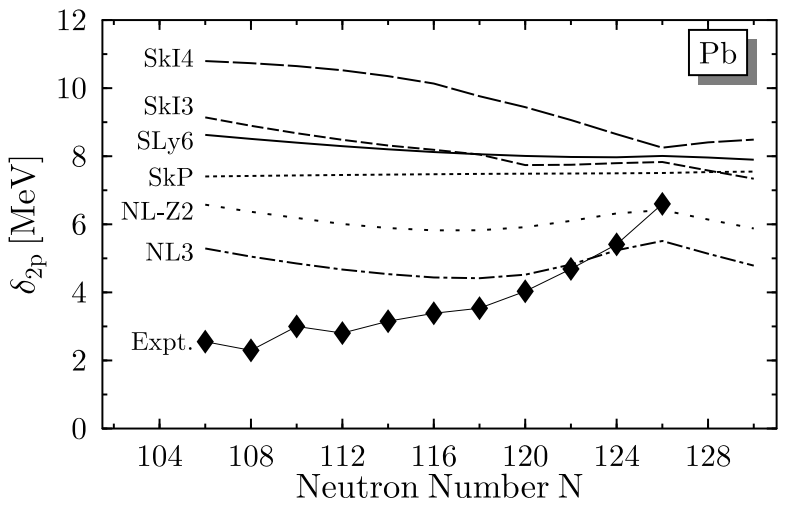

Fig. 1. Two-proton gap parameter $\delta_{2 p}$ (2) for the chain of $\mathrm{Pb}$ isotopes obtained in several spherical mean-field models. Experimental values are marked with filled diamonds.

\section{Results}

\subsection{Spherical calculations}

We start with a presentation of purely spherical calculations. By constraining the shape to be spherical, one can dramatically reduce polarization effects. Consequently, the assumptions behind the Koopman's theorem can be met, and the clear correspondence between $\delta_{2 p}$ and single-particle energies emerges. Figure 1 shows the two-proton shell gap parameter $\delta_{2 p}$ along the chain of $\mathrm{Pb}$ isotopes. The experimental data are compared with results of spherical meanfield calculations. While the experimental values decrease monotonously when going from ${ }^{208} \mathrm{~Pb}$ towards the protondrip line, theoretical results show a very different trend. Namely, $\delta_{2 p}$ slightly decreases with $Z$. The corresponding spherical single-proton energies in SkI3 are displayed in fig. 2. It is seen that the $Z=82$ shell gap stays large for all

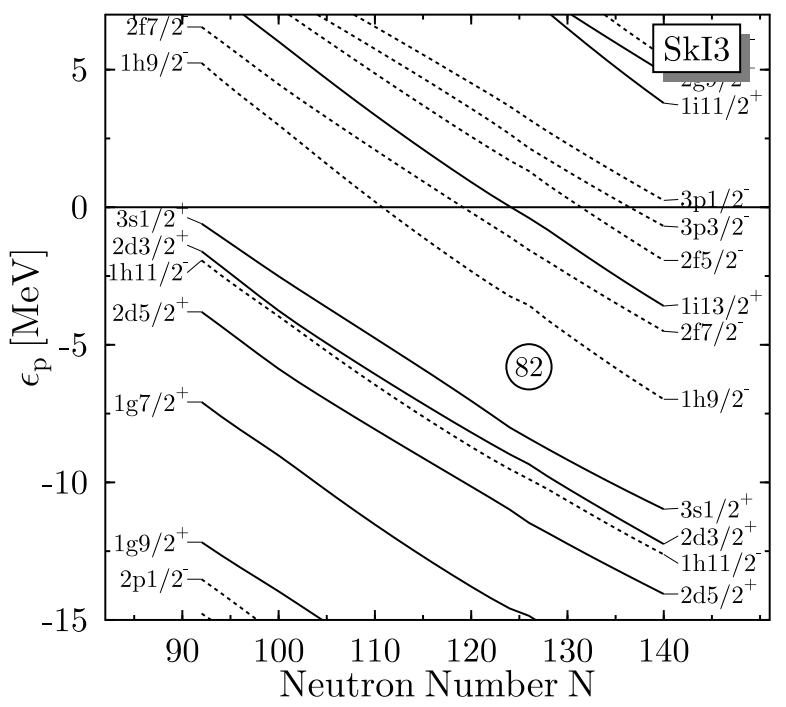

Fig. 2. Self-consistent single-proton energies $\epsilon_{k}$ for the chain of $\mathrm{Pb}$ isotopes calculated with the spherical SkI3 model. Other effective interactions give similar results. See ref. [30] for a comparison of calculated and experimental spectra for ${ }^{208} \mathrm{~Pb}$.

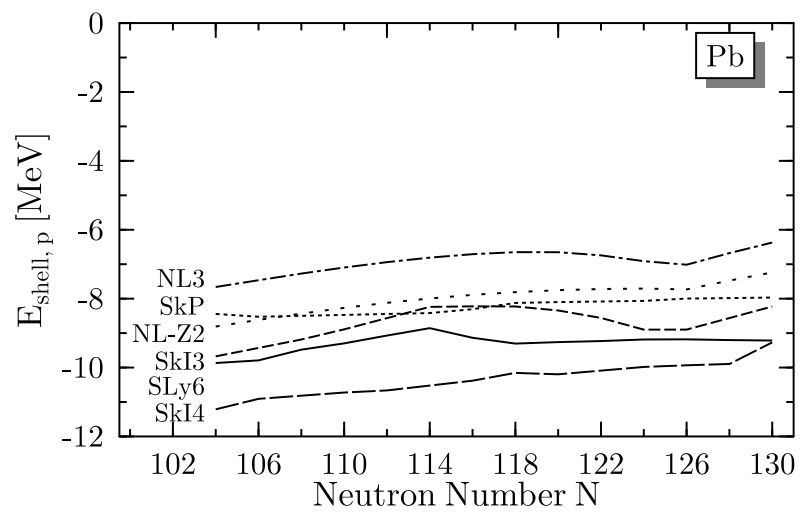

Fig. 3. Spherical proton shell correction for the chain of $\mathrm{Pb}$ isotopes extracted from self-consistent calculations.

the $\mathrm{Pb}$ isotopes considered, and it grows slightly when approaching the proton drip line. (Other parameterizations employed in our study give similar results.) This result is consistent with fig. 1: $\delta_{2 p}$ reproduces twice the shell gap seen in the spectrum of $\epsilon_{k}$. This demonstrates that the idea beyond the two-nucleon shell gap as a signature of shell closures is correct, provided the mean field does not change much between the nuclei involved in its evaluation. The restriction to spherical calculations enforces this feature.

The stability of the $Z=82$ shell is confirmed when inspecting the proton shell correction energy $E_{\text {shell,p }}$ extracted from our self-consistent calculations; see fig. 3. There are some differences among the various forces as far as the actual values are concerned. For instance, RMF models generally yield smaller shell correction than SHF models. The common feature seen in fig. 3 is that all the models predict the same trend, namely that the magnitude of the proton shell correction is slightly increasing when going proton-rich. One can thus say that all the indicators of shell effects considered in our study, i.e., single-particle energies, shell corrections, and $\delta_{2 p}$, give a consistent picture of a stable $Z=82$ shell for all neutron-deficient $\mathrm{Pb}$ isotopes. Contrary to what may be suggested by the experimental plot of $\delta_{2 p}$, the proton magic gap does not decrease, but slightly increases when approaching the proton drip line.

\subsection{Deformed calculations}

It is well known that shape coexistence is a common feature in neutron-deficient $\mathrm{Hg}, \mathrm{Pb}$, and $\mathrm{Po}$ nuclei, and that most of the $\mathrm{Hg}$ and Po nuclei have deformed ground states [32 33]. Figure 4 shows the calculated deformation energies $\Delta E$ (defined as a difference between ground-state binding energies obtained in deformed and spherical calculations) and quadrupole deformations $\beta_{2}$ for even-even $\mathrm{Hg}$, $\mathrm{Pb}$, and $\mathrm{Po}$ isotopic chains. Most nuclei have deformed ground states with an energy gain of several MeV. The potential energy surfaces calculated in this region exhibit several competing minima, often leading to shape coexistence and configuration mixing, see e.g., refs. 34,35. 


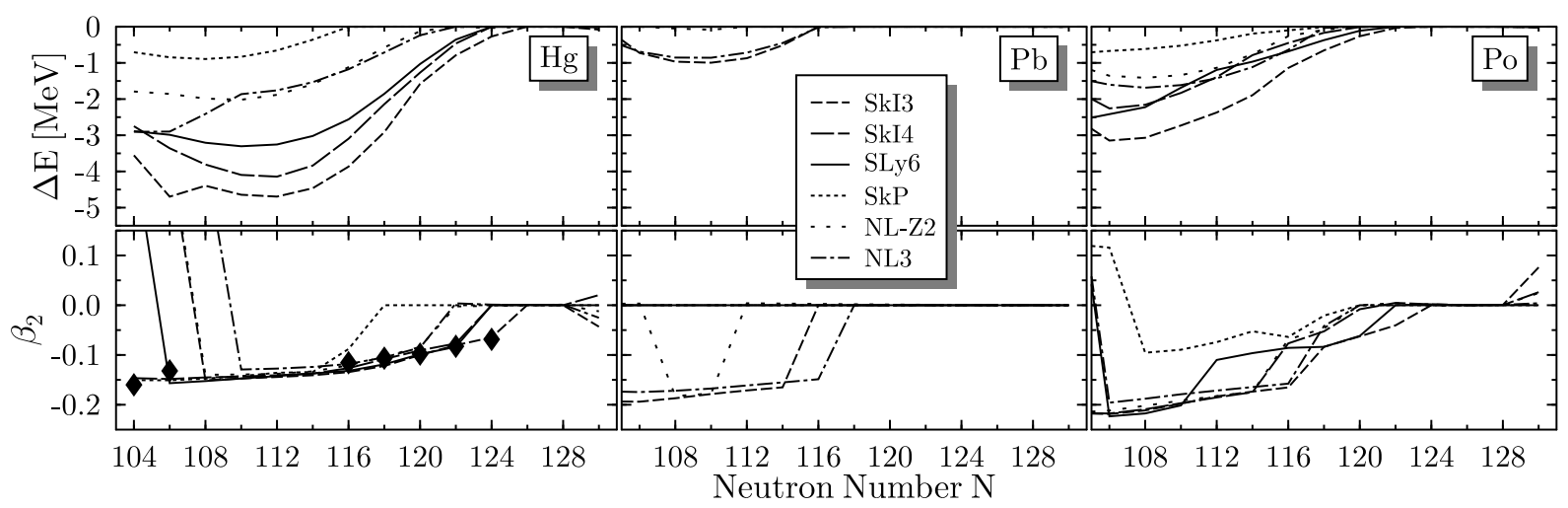

Fig. 4. Calculated deformation energy $\Delta E$ (top) and associated quadrupole deformation $\beta_{2}=4 \pi\left\langle r^{2} Y_{20}\right\rangle /\left(3 A r_{0}^{2}\right)$ with $r_{0}=1.2 A^{1 / 3} \mathrm{fm}$ (bottom) for the ground states of even-even $\mathrm{Hg}, \mathrm{Pb}$, and $\mathrm{Po}$ isotopes. Experimental quadrupole deformations (filled diamonds) are taken from ref. [49].

In fact, the question if whether there were strongly deformed nuclei near the magic proton shell $Z=82$ was already raised as early as 1972 [37,38]. Experimentally, the even-even ${ }^{176-190} \mathrm{Hg}$ isotopes have oblate ground states but also prolate excited states 36$]$. The data on excitation spectra and charge radii for the $\mathrm{Pb}$ isotopes, on the other hand, are consistent with the assumption that the ground states down to $N=90$ are spherical [33, 39, 40], although one has to be aware that the potential landscape for $\mathrm{Pb}$ also becomes softer for proton-rich isotopes. As a result, coexisting oblate and prolate minima show up [32,35], the most spectacular being the multitude of coexisting structures in ${ }^{186} \mathrm{~Pb}[41$. A systematic analysis of available excitation data in Po isotopes demonstrates that collectivity increases rapidly in neutron-deficient isotopes 33, 42 .

When deformation effects are taken into account, the behavior of $\delta_{2 p}$ changes dramatically. As shown in fig. 5 , all our models predict gradually decreasing $\delta_{2 p}$ towards the proton drip line in accordance with experiment. The crucial point is that one deals with systems where the mean field is extremely sensitive to small changes in nucleon number. These rearrangement effects strongly affect

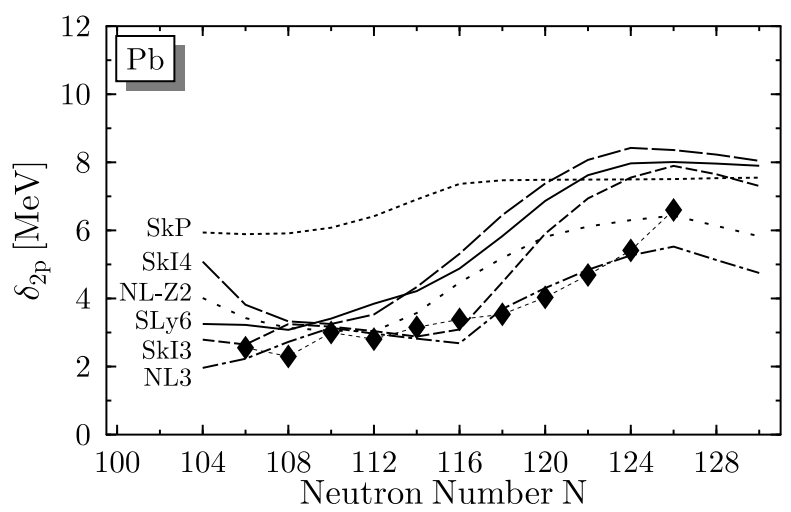

Fig. 5. Two-proton gap parameter $\delta_{2 p}$ (2) for the chain of $\mathrm{Pb}$ isotopes obtained in several deformed mean-field models. Experimental values are marked with filled diamonds. the behavior of $\delta_{2 p}$ and mask the presence of the magic $Z=82$ proton shell; it is nuclear deformation that explains the apparent discrepancy seen in fig. $\mathbb{1}$.

Complementary information on shell effects can be drawn from $\alpha$-decay studies. An analysis of reduced $\alpha$ widths and $Q_{\alpha}$ values of nuclei around ${ }^{182} \mathrm{~Pb}$ suggests a weakening of the $Z=82$ shell effect [20]. However, since $Q_{\alpha}$ values are obtained from a finite-difference formula similar to $\delta_{2 p}$, experimental systematics $Q_{\alpha}$ reflect deformation effects and the structural change of ground states between the isotones of $\mathrm{Hg}, \mathrm{Pb}$, and $\mathrm{Po}$. Moreover, $\alpha$-decay hindrance factor systematics can be understood assuming the stability of the spherical $Z=82$ shell at the very proton-rich side 43,44 and can be described in the language of shape mixing 43.45$]$.

The rich structure of potential landscapes in the region of proton-rich $\mathrm{Pb}$ nuclei provides a challenging benchmark for theoretical modeling. The quantitative description of all details is beyond the abilities of current self-consistent mean-field models and, because of the presence of lowlying intruder states, also requires the use of sophisticated techniques which incorporate configuration mixing effects. Small shifts in single-particle levels or changes in the parameterization of pairing interaction do influence relative positions of coexisting minima. Therefore, many forces miss the exact location of the onset of deformation for the $\mathrm{Hg}$ isotopes, see fig. A and 46]. Some forces (RMF and SkI3) even predict deformed ground states in some $\mathrm{Pb}$ isotopes - in contradiction with experiment. We have seen in fig. A that the inclusion of deformation improves the agreement of theory with experiment for $\delta_{2 p}$. Still, some discrepancies remain. Note that all SHF forces already overestimate $\delta_{2 p}$ for the doubly magic ${ }^{208} \mathrm{~Pb}$, although this nucleus is spherical, as well as its neighboring nucleus. The simplest excuse is that the single-particle states in ${ }^{208} \mathrm{~Pb}$ are not perfectly described by the models (see, e.g., 30]). However, another important source of discrepancy are correlations beyond the mean field. Their influence on the two-neutron separation energies around ${ }^{208} \mathrm{~Pb}$ has been studied using the generator coordinate method (GCM) in ref. 477. The important outcome is 
that the quadrupole correlations decrease the two-neutron shell gap at the shell closure. A similar effect can be expected for the two-proton shell gap parameter. The effect of quadrupole correlations on $\delta_{2 p}$ is probably largest in the transitional region of the onset of static deformation around $N=120$ where also the discrepancy between experiment and our mean-field calculations is largest. Configuration mixing effects in proton-rich $\mathrm{Pb}$ isotopes were studied in ref. 48], but were aimed at excitation energies, and not mass systematics (see also recent GCM work [50 ).

\section{Summary}

Finite-difference binding-energy indicators such as $\delta_{2 q}$ or $Q_{\alpha}$ lose their validity as signatures of shell closures as soon as they are used in a region where the structure of nuclear ground states is rapidly changing. Such changes of nuclear ground state configurations with $Z$ and $N$ explain qualitatively the systematics of experimental $\delta_{2 p}$ and $Q_{\alpha}$ in the neutron-deficient $\mathrm{Pb}$ region. In particular, our calculations do not support previous speculations about quenching of the $Z=82$ shell in the neutron-deficient $\mathrm{Pb}$ isotopes.

It is important, however, to remember that the complex structure of nuclei from shape-coexisting regions limits their description in terms of mean-field models. The presence of low-lying intruder states having different shapes requires the use of theories which can account for configuration mixing effects. Therefore, the whole notion of a "shell gap", which originates from the mean-field theory, can be questionable in such situations. In general, one has to be aware that the usual signature of spherical shell closures might not be robust when going to exotic nuclei far from stability in which correlations play an important role.

\section{Acknowledgements}

Discussions with H. Geissel, H. Grawe, G. Münzenberg, Ch. Scheidenberger, and H. Wollnik are gratefully acknowledged. This work was supported in part by Bundesministerium für Bildung und Forschung (BMBF), Project No. 06 ER 808, by Gesellschaft für Schwerionenforschung (GSI), and by the U.S. Department of Energy under Contract Nos. DE-FG02-96ER40963 (University of Tennessee), DEFG05-87ER40361 (Joint Institute for Heavy Ion Research), and DE-AC05-00OR22725 with UT-Battelle, LLC (Oak Ridge National Laboratory).

\section{References}

1. D. Guillemaud-Mueller, C. Detraz, M. Langevin, F. Naulin, M. de Saint-Simon, C. Thibault, F. Touchard, and M. Epherre, Nucl. Phys. A426, 37 (1984).

2. D. J. Vieiera, J. M. Wouters, K. Vaziri, R. H. Kraus Jr., H. Wollnik, G. W. Butler, F. K. Wohn, and A. H. Wapstra, Phys. Rev. Lett. 57, 3253 (1986).
3. N. A. Orr, W. Mittig, L. K. Fifield, M. Lewitowicz, E. Plagnol, Y. Schutz, Zhan Wen Long, L. Bianchi, A. Gilibert, A. V. Belozyorov, S. M. Kukyanov, Yu. E. Penionzhkevich, A. C. C. Villari, A. Cunsolo, A. Foti, G. Audi, C. Stephan, and L. Tassan-Got, Phys. Lett. B258, 29 (1991).

4. X. G. Zhou, X. L. Tu, J. M. Wouters, D. J. Vieira, K. E. G. Löbner, H. L. Seifert, Z. Y. Zhou, and G. W. Butler, Phys. Lett. B260, 285 (1991).

5. T. Glasmacher, B. A. Brown, M. J. Chromik, P. D. Cottle, M. Fauerbach, R. W. Ibbotson, K. W. Kemper, D. J. Morrissey, H. Scheit, D. W. Slenicka, and M. Steiner, Phys. Lett. B395, 163 (1997).

6. K.-L. Kratz, V. Harms, A. Wöhr, and P. Möller, Phys. Rev. C 38, 278 (1988).

7. T. Kautzsch, W. B. Walters, M. Hannawald, K.-L. Kratz, V. I. Mishin, V. N. Fedoseyev, W. Böhmer, Y. Jading, P. Van Duppen, B. Pfeiffer, A. Wöhr, P. Möller, I. Klöckl, V. Sebastian, U. Köster, M. Koizumi, J. Lettry, H. L. Ravn, and the ISOLDE Collaboration, Eur. Phys. J. A9, 201 (2000).

8. B. Pfeiffer, K.-L. Kratz, and F.-K. Thielemann, Nucl. Phys. A357, 235 (1997).

9. J. Dobaczewski, I. Hamamoto, W. Nazarewicz, and J. A. Sheikh, Phys. Rev. Lett. 72, 981 (1994).

10. G. A. Lalazissis, D. Vretenar, W. Poeschl, and P. Ring, Phys. Lett. B418, 7 (1998).

11. T. R. Werner, J. A. Sheikh, W. Nazarewicz, M. R. Strayer, A. S. Umar, and M. Misu, Phys. Lett. B333, 259 (1994).

12. G. A. Lalazissis, D. Vretanar, P. Ring, M. V. Stoitsov, and L. M. Robledo, Phys. Rev. C 60, 014310 (1999).

13. S. Péru, M. Girod, and J. F. Berger, Eur. Phys. J. A9, 35 (2000).

14. P.-G. Reinhard, D. J. Dean, W. Nazarewicz, J. Dobaczewski, J. A. Maruhn, and M. R. Strayer, Phys. Rev. C 60, 014316 (1999).

15. R. Broda, B. Formal, W. Królas, T. Pawlat, D. Bazzacco, S. Lunardi, C. Rossi-Alvarez, R. Menegazzo, G. de Angelis, B. Bednarczyk, J. Rico, D. De Acuña, P. J. Daly, R. H. Mayer, M. Sferrazza, H. Grawe, K. H. Maier, and R. Schubart, Phys. Rev. Lett. 74, 868 (1995).

16. H. Grawe, M. Górska, C. Fahlander, M. Palacz, F. Nowacki, E. Caurier, J. M. Daugas, M. Lewitowicz, M. Sawicka, R. Grywacz, K. Rykaczewski, O. Sorin, S. Leenhardt, and F. Azaiez, Proc. of the RIKEN Symposium "Shell Model 2000", to be published in Nucl. Phys. A, GSI preprint 2000-17.

17. P.-G. Reinhard, M. Bender, T. Bürvenich, C. Reiss, J. A. Maruhn, and W. Greiner, Proceedings of the "International Symposium on Models and Theories of the Nuclear Mass", RIKEN, Japan, July 19-23 1999, N. Tajima and S. Yamaji [eds.], RIKEN Review 26, 23 (2000).

18. T. Radon, H. Geissel, G. Münzenberg, B. Franzke, Th. Kerscher, F. Nolden, Yu. N. Novikov, Z. Patyk, C. Scheidenberger, F. Attallah, K. Beckert, T. Beha, F. Bosch, H. Eickhoff, M. Falch, Y. Fujita, M. Hausmann, F. Herfurth, H. Irnich, H. C. Jung, O. Klepper, C. Kozhuharov, Yu. A. Litvinov, K. E. G. Löbner, F. Nickel, H. Reich, W. Schwab, B. Schlitt, M. Steck, K. Sümmerer, T. Winkler, and H. Wollnik, Nucl. Phys. A677, 75 (2000).

19. Yu. N. Novikov, F. Attallah, F. Bosch, M. Falch, H. Geissel, M. Hausmann, Th. Kerscher, O. Klepper, H.-J. Kluge, C. Kozhuharov, Yu. A. Litvinov, K. E. G. Löbner, G. Münzenberg, Z. Patyk, T. Radon, C. Scheidenberger, A. H. Wapstra, H. Wollnik, preprint, 2000. 
20. K. S. Toth, C. R. Bingham, J. C. Batchelder, L.T. Brown, L. F. Conticchio, C.N. Davids, R. J. Irvine, D. Seweryniak, D. M. Moltz, W. B. Walters, J. Wauters, and E. F. Zganjar, Phys. Rev. C 60, 011302 (1999).

21. M. Brack, J. Damgård, A. S. Jensen, H. C. Pauli, V. M. Strutinsky, and C. Y. Wong, Rev. Mod. Phys. 44, 320 (1972).

22. V. Bernard and Nguyen Van Giai, Nucl. Phys. A348, 75 (1980).

23. K. Rutz, M. Bender, P.-G. Reinhard, J. A. Maruhn, and W. Greiner, Nucl. Phys. A634, 67 (1998).

24. M. Bender, W. Nazarewicz, and P.-G. Reinhard, Phys. Lett. B515, 42 (2001).

25. A. T. Kruppa, M. Bender, W. Nazarewicz, P.-G. Reinhard, T. Vertse, and S. Cwiok, Phys. Rev. C 61, 034313 (2000).

26. J. Dobaczewski, H. Flocard, and J. Treiner, Nucl. Phys. A422, 103 (1984).

27. E. Chabanat, P. Bonche, P. Haensel, J. Meyer, and R. Schaeffer, Nucl. Phys. A635, 231 (1998); Nucl. Phys. A643, 441(E) (1998).

28. P.-G. Reinhard and H. Flocard, Nucl. Phys. A584, 467 (1995).

29. G. A. Lalazissis, J. König, and P. Ring, Phys. Rev. C 55, 540 (1997).

30. M. Bender, K. Rutz, P.-G. Reinhard, J. A. Maruhn, and W. Greiner, Phys. Rev. C 60, 034304 (1999).

31. M. Bender, K. Rutz, P.-G. Reinhard, and J. A. Maruhn, Eur. Phys. J. A8 59 (2000).

32. J. L. Wood, K. Heyde, W. Nazarewicz, M. Huyse, and P. van Duppen, Phys. Rep. 215, 101 (1992).

33. R. Julin, K. Helariutta, and M. Muikku, J. Phys. (London) G27, R109 (2001).

34. F. R. May, V. V. Pashkevich, and S. Frauendorf, Phys. Lett. 68B, 113 (1977).

35. W. Nazarewicz, Phys. Lett. 305B, 195 (1993).

36. K. Helariutta, M. Muikku, J. F. C. Cocks, P. Jones, R. Julin, S. Juutinen, H. Kankaanpaa, H. Kettunen, P. Kuusiniemi, M. Leino, P. Rahkila, A. Savelius, W. H. Trzaska, J. Uusitalo, P. T. Greenlees, and R. D. Page, Acta Phys. Pol. B30, 1267 (1999).

37. A. Faessler, U. Götz, B. Slavov, and T. Ledergerber, Phys. Lett. 39B, 579 (1972).

38. M. Cailliau, J. Letessier, H. Flocard, and P. Quentin, Phys. Lett. 46B, 11 (1973).

39. D. G. Jenkins, M. Muikku, P. T. Greenlees, K. Hauschild, K. Helariutta, P. M. Jones, R. Julin, S. Juutinen, H. Kankaanpaa, N.S. Kelsall, H. Kettunen, P. Kuusiniemi, M. Leino, C. J. Moore, P. Nieminen, C. D. O'Leary, R. D. Page, P. Rakhila, W. Reviol, M. J. Taylor, J. Uusitalo, and R. Wadsworth, Phys. Rev. C 62, 021302 (2000).

40. K. Heyde, C. De Coster, P. Van Duppen, M. Huyse, J. L. Wood, and W. Nazarewicz, Phys. Rev. C 53, 1035 (1996).

41. A. N. Andreyev, M. Huyse, P. Van Duppen, L. Weissman, D. Ackermann, J. Gerl, F. P. Hessberger, S. Hofmann, A. Kleinbohl, G. Münzenberg, S. Reshitko, C. Schlegel, H. Schaffner, P. Cagarda, M. Matos, S. Saro, A. Keenan, C. Moore, C. D. O'Leary, R. D. Page, M. Taylor, H. Kettunen, M. Leino, A. Lavrentiev, R. Wyss, and K. Heyde, Nature (London) 405, 430 (2000).

42. W. Younes and J.A. Cizewski, Phys. Rev. C 55, 1218 (1997).

43. J. Wauters, N. Bijnens, P. Dendooven, M. Huyse, H.Y. Hwang, G. Reusen, J. von Schwarzenberg, P. Van Duppen,
R. Kirchner, E. Roeckl, and the ISOLDE Collaboration, Phys. Rev. Lett. 72, 1329 (1994).

44. N. Bijnens, J. G. Correia, M. Huyse, H. Y. Hwang, R. Kirchner, G. Reusen, E. Roeckl, P. Van Duppen, J. von Schwarzenberg, J. Wauters, and the ISOLDE Collaboration, Phys. Scr. T56, 110 (1995)

45. J. D. Richards, T. Berggren, C. R. Bingham, W. Nazarewicz, and J. Wauters, Phys. Rev. C 56, 1389 (1997).

46. P.-G. Reinhard, C. Reiss, M. Bender, T. Bürvenich, T. Cornelius, and J. A. Maruhn, Hyperfine Interactions 127, 13 (2000).

47. P.-H. Heenen, A. Valor, M. Bender, P. Bonche, and H. Flocard, Eur. Phys. J. A11, 393 (2001).

48. J. Meyer, P. Bonche, M. S. Weiss, J. Dobaczewski, H. Flocard, and P.-H. Heenen, Nucl. Phys. A588, 597 (1995)

49. S. Raman, C. W. Nestor Jr., and P. Tikkanen, At. Data Nucl. Data Tables, 78, 1 (2001).

50. R. R. Chasman, J. L. Egido, and L. M. Robledo, Phys. Lett. B513, 325 (2001). 